\title{
Microstructures, mechanical behavior, cellular response, and hemocompatibility of bulk ultrafine-grained pure tantalum
}

\author{
F. L. Nie, ${ }^{1,2}$ Y. F. Zheng, ${ }^{1,3}$ Y. Wang, ${ }^{4}$ J. T. Wang ${ }^{4}$ \\ ${ }^{1}$ State Key Laboratory for Turbulence and Complex System and Department of Materials Science and Engineering, College of \\ Engineering, Peking University, Beijing 100871, China \\ ${ }^{2}$ Center for Medical Device Evaluation, SFDA, Beijing 100044, China \\ ${ }^{3}$ Center for Biomedical Materials and Tissue Engineering, Academy for Advanced Interdisciplinary Studies, Peking University, \\ Beijing 100871, China \\ ${ }^{4}$ Department of Materials Science and Engineering, School of Materials Science and Engineering, Nanjing University of \\ Science and Technology, Nanjing 210094, China
}

Received 12 August 2012; revised 1 April 2013; accepted 6 June 2013

Published online 2 August 2013 in Wiley Online Library (wileyonlinelibrary.com). DOI: 10.1002/jbm.b.32998

\begin{abstract}
Bulk ultrafine-grained (UFG) pure Ta had been successfully prepared by equal channel angular pressing (ECAP) technique till eight passes. The 1st, 2nd, 4th, and 8th ECAPed Ta samples were investigated in the current study, with the Oth ECAPed Ta sample as the microcrystalline counterpart control. The microstructure and grain size distribution were characterized by X-ray diffractometer patterns, scanning electron microscopy, and transmission electron microscopy analysis by means of histogram. Although the mechanical behavior of all the experimental samples were analyzed through uniaxial tensile measurement and microhardness test, in vitro biological interactions onto the substrates such as protein adsorption, cellular responses derived from different types of cell lines, and the activity of erythrocyte and platelets were further evaluated and specifically assessed by bicinchoninic acid assay, enzyme-linked immunosorbent assay, and the method of colorimetric reading. A superior
\end{abstract}

percentage of protein adsorption can be observed on the substrate of the UFG 8th ECAPed Ta (around 90\%), even above those on the tissue culture plate (control) and the other ECAPed Ta samples. Furthermore, the UFG 8th ECAPed Ta shows no cytotoxic within 4 days culture when incubated with the murine fibroblast cell lines (L929). In addition, a priority order in the growth of endothelial cells (ECV304) other than vascular smooth muscle cells was observed in the case of the UFG 8th ECAPed Ta. In terms of hemolysis rate and adhered platelets (both the amount and the individual morphology), an evolutionary outcome of preferentially enhanced hemocompatibility can be concluded for the case of the UFG 8th ECAPed Ta. (c) 2013 Wiley Periodicals, Inc. J Biomed Mater Res Part B: Appl Biomater, 102B: 221-230, 2014.

Key Words: bulk ultrafine-grained Ta, ECAP, cellular responses, hemocompatibility

How to cite this article: Nie FL, Zheng YF, Wang Y, Wang JT. 2014. Microstructures, mechanical behavior, cellular response, and hemocompatibility of bulk ultrafine-grained pure tantalum. J Biomed Mater Res Part B 2014;102B:221-230.

\section{INTRODUCTION}

Pure tantalum (abbreviated as Ta), which is a representative heavy-metal element, has been commonly used as sutures, surgical plates/fixations, or hip joint replacements over 50 years application history. ${ }^{1,2}$ under the restricted biomedical regulations for its good thermal stability (up to $300^{\circ} \mathrm{C}$ ), corrosion resistance (higher corrosion potential than pure $\mathrm{Ti}$ or $\mathrm{Cr}){ }^{3}$ and high-fluoroscopic visibility (high density). ${ }^{4}$ Publications or reports on pure Ta-concentrated biological responses, including in vitro bone-like apatite forming capability, ${ }^{5}$ in vivo little adverse effects, a covering of collagen and myofibroblastic cells in foil, wire or mesh implantation in soft tissues ${ }^{3}$ and no metallic dissolution in local, systemic, and remote organs reveal its well-behaved biocompatibility with good healing even in the course of the osseointegration process. ${ }^{6-8}$ However, the insufficient mechanical strength and the minimum gap between yield and tensile strength may lead rapid and undesirable premature failure once over necking in the loading deployment, ${ }^{4}$ which is regarded as the mainly discovered shortage for the present. Moreover, no Food and Drug Administration-approved bare Ta bulk as cardiovascular stents or vessels are in general use up to now for the serious consideration of its potential risk of enhanced recoiling and neointimal formation, even an

Correspondence to: Y.-F. Zheng; e-mail: yfzheng@pku.edu.cn or J.T. Wang; e-mail: jtwang@mail.njust.edu.cn

Contract grant sponsor: National Basic Research Program of China (973 Program); contract grant numbers: 2012 CB619102 and 2012CB619100

Contract grant sponsor: National Science Fund for Distinguished Young Scholars; contract grant number: 51225101

Contract grant sponsor: Research Fund for the Doctoral Program of Higher Education; contract grant number: 20100001110011

Contract grant sponsor: National Natural Science Foundation of China; contract grant number: 31170909

Contract grant sponsor: Natural Science Foundation of Heilongjiang Province; contract grant number: ZD201012 
TABLE I. Chemical Compositions of Pure Ta (wt \%)

\begin{tabular}{|c|c|c|c|c|c|c|c|c|c|c|c|c|}
\hline Element & $\mathrm{Nb}$ & $\mathrm{Ti}$ & W & Mo & $\mathrm{Fe}$ & $\mathrm{Ni}$ & $\mathrm{Si}$ & C & $\mathrm{N}$ & $\mathrm{H}$ & $\mathrm{O}$ & $\mathrm{Ta}$ \\
\hline Content & 0.02 & 0.01 & 0.01 & 0.01 & 0.02 & 0.02 & 0.018 & 0.04 & 0.01 & 0.008 & 0.02 & Bal. \\
\hline
\end{tabular}

accelerated endothelialization rate occurs with the increment of the Ta concentration as reported in literature. ${ }^{9}$

Many ways like foaming and surface treatment were adopted to regulate the adjustment of pure $\mathrm{Ta}^{7,10-12}$ However, much high melting point and the conceivable mismatch between surface and substrates limits the practical application of the methods mentioned above. Then, it was not until the discovery of the technique of severe plastic deformation, which was proved to be an economical and feasible way onto pure Ta due to its good workability, would the problematic issues of improvement on the microstructure together with the performances be solved perfectly even free from the interface dilemma. Apparently, the nature of refinement in the microstructural grain sizes and textures would prospectively enhance the strengthening very well. Furthermore, from recent literatures, bulk ultrafine-grained (UFG) metals such as pure $\mathrm{Cu}, \mathrm{Ti}$, and $\mathrm{Zr}$ with nanoscaled grains or microtextures and controllable deficiency existence reportedly exhibit enhanced properties in physical, chemical, or biological fundamentals.

Herein, the process of nanotechnology-induced severe plastic deformation is used to produce bulk UFG Ta samples until eight passes equal channel angular pressing (ECAP) in the present investigation, with the 0 th, 1st, 2nd, and 4th ECAPed specimens as the counterparts. After the repetitive deformation and accumulative shear in the process of ECAP technique, microscale grains turn into small pieces under high-density dislocation cut within submicron meters, even UFG clusters. A further design of experimental measurements on materials characterization, mechanical behavior, protein bioaffinity, cellular interactions, and hemocompatibility responses were specifically studied to explore its biocompatibility and the corresponding correlation between the evolutionary microstructures and their performances.

\section{MATERIALS AND METHODS}

\section{Sample preparation and fundamental physiochemical characterization}

Pure Ta (initially provided by the manufacturer of Xi'an Yaxin, with the chemical composition details in Table I) of eight passes were fabricated via $\mathrm{Bc}$ route (which represents that the sample rotates by $90^{\circ}$ along the longitudinal axis in clockwise during the extrusion interval $)^{13}$ of ECAP technique. The 0th, 1st, 2nd, 4th, and 8th ECAPed samples were selected and investigated here in the present study. All the samples were sliced into discs of $10 \times 10 \times 1 \mathrm{~mm}^{3}$ by electrical discharge wire cutting and then mechanically polished to $2000 \#$ sand paper. All the samples were ultrasonically cleaned in acetone, absolute ethanol, and distilled water and then quickly dried in the open air before any measurement. X-ray diffractometer (XRD, Rigaku DMAX 2400) using $\mathrm{Cu} \mathrm{K \alpha}$ radiation was used for phase identification. Optical micros- copy (Olympus BX 51M) was used for microstructural observation of all the pure Ta samples after etching in a mixture solution of two part nitric acid and one part $60 \%$ ammonium fluoride in a standard way. The tensile tests (the specimen of $1.5 \times 1.5 \times 40 \mathrm{~mm}^{3}$ ) were performed at a strain rate of $0.01 \mathrm{~s}^{-1}$ with an Instron 3366 universal test machine under ambient temperature. Bright field of transmission electron microscopy (TEM; FEI Tecnai G2 T20) and selected area electron diffraction (SAED) were measured, and the dimple samples were prepared by ion milling (Gatan 691) at a slow scan rate in incident gradient angles (from $10^{\circ}$ to $4^{\circ}$ ). Microhardness was measured using a Vickers diamond pyramidal indenter (HMV-2T, Shimadzu) under a load of $200 \mathrm{~g}$ for $10 \mathrm{~s}$. For the extraction preparation in the cell test, all the samples were sterilized in the autoclave at $121^{\circ} \mathrm{C}$ for $30 \mathrm{~min}$.

A three-electrode cell was used for electrochemical measurements. The counterelectrode was made of platinum, and the reference electrode was saturated calomel electrode. The exposed area of the working electrode to the solution was $0.1256 \mathrm{~cm}^{2}$. Open circuit potential (OCP, till $7200 \mathrm{~s}$ ) and polarization curve (with the scan ratio of $0.001 \mathrm{~V} / \mathrm{s}$ ) in simulated body fluid (SBF) were all performed on an electrochemical workstation (CHI660C, China) at the temperature of $37^{\circ} \mathrm{C}$, stored in the water bath.

The immersion test was performed according to ASTM $^{14}$ in SBF. Experimental samples $\left(10 \times 10 \times 1 \mathrm{~mm}^{3}\right)$ were immersed in $40 \mathrm{~mL}$ solutions, and the temperature was kept at $37^{\circ} \mathrm{C}$ by water bath. The inductively coupled plasma atomic emission spectrometry (Leeman, Profile ICP-AES) was used to measure the concentrations of $\mathrm{Ta}, \mathrm{Fe}$, and $\mathrm{Ni}$ element ions, which might be dissolved from the alloy samples.

\section{Protein adsorption}

Bovine serum albumin (Baosai Biotechnology, Beijing) was used as a standardized model protein. One hundred microliters of protein solution $(0.2 \mathrm{mg} / \mathrm{mL}$ protein/saline $)$ was pipetted onto the surface of the experimental substrate, with the tissue culture plate (TCP; polystyrene) as control and saline as blank. After incubation for 2, 4, and $24 \mathrm{~h}$ at $37^{\circ} \mathrm{C}$, nonadherent proteins were removed and mixed with micro-bicinchoninic acid at $37^{\circ} \mathrm{C}$ for $30 \mathrm{~min}^{15}$ The amount of the removed albumin, as well as the total amount of albumin inoculated, was quantified using a microplate reader at $570 \mathrm{~nm}$. The rate of albumin adsorption was calculated as the percentage of albumin adsorbed to tantalum surfaces relative to the total amount.

\section{Cytotoxicity and cellular responses}

Indirect cytotoxicity test of extraction via MTT assay was performed according to a standard procedure described 


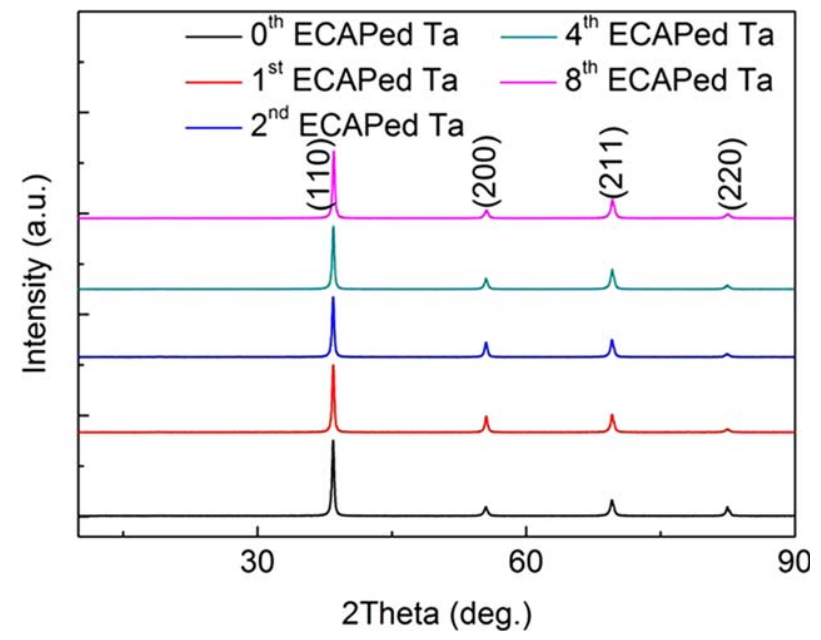

FIGURE 1. XRD spectra of the 0th, 1st, 2nd, 4th, and 8th ECAPed Ta. [Color figure can be viewed in the online issue, which is available at wileyonlinelibrary.com.]

using cells from American type culture collection (ATCC): murine fibroblast cells (L929), vascular smooth muscle cells (VSMCs), and endothelial cells (ECV304). Cells were directly seeded onto the substrates of all the experimental Ta samples to observe the situation of cellular attachment, spread, and morphologies. Extracts were prepared using Dulbecco's modified eagle's medium (DMEM) serum-free medium as the extraction medium with the surface area of extraction medium ratio being $3 \mathrm{~cm}^{2} / \mathrm{mL}^{16}$ in a humidified incubator with $5 \% \mathrm{CO}_{2}$ at $37^{\circ} \mathrm{C}$ for $72 \mathrm{~h}$. DMEM here consists of $10 \%$ fetal bovine serum, $100 \mathrm{U} / \mathrm{mL}$ penicillin, and $100 \mu \mathrm{g} / \mathrm{mL}$ streptomycin. Negative controls of DMEM-cultured cells and positive controls of DMSO-added cells were involved. A cell density of $5 \times 10^{4} \mathrm{~mL}^{-1}$ was seeded directly on the substrate of different samples in the 24-well plate. Optical density (OD) values from cells in the well plate were detected at $570 \mathrm{~nm}$ with a reference wavelength of $630 \mathrm{~nm}$ by microplate reader (Bio-RAD680). The percentage of viability is obtained by the formula as follows:

$$
\text { Cell viability }=\frac{O D(\text { test })}{O D(\text { negative control })} \times 100 \%
$$

\section{Hemolysis rate and adhered platelets}

Peripheral blood was obtained from healthy donors under consent and ethical allowance. Diluted blood was prepared with normal saline (4:5 ratio by volume) by containing sodium citrate (3.8 wt \%) in the ratio of 9:1. Experimental samples were immersed in a $10-\mathrm{mL}$ saline tube preincubated at $37^{\circ} \mathrm{C}$ for $30 \mathrm{~min}$, followed by $60 \mathrm{~min}$ incubation after the addition of $0.2 \mathrm{~mL}$ diluted blood into this tube, with physiological saline solution and deionized water as negative and positive controls, respectively. Then, all the tubes were centrifuged for $5 \mathrm{~min}$ at $3000 \mathrm{rpm}$, and the supernatant was carefully taken out to the cuvette for spectroscopic analysis at $545 \mathrm{~nm}$. The hemolysis was calculated based on the average of three replicates.
Hemolysis $=\frac{O D(\text { test })-O D(\text { negative control })}{O D(\text { positive control })-O D(\text { negative control })} \times 100 \%$

Platelet-rich plasma was prepared by centrifuging the whole blood for $10 \mathrm{~min}$ at a rate of $1000 \mathrm{rpm} / \mathrm{min}$. Then, it was dripped onto the sample surface and incubated for $1 \mathrm{~h}$ at $37^{\circ} \mathrm{C}$. Thereafter, phosphate-buffered saline (PBS) was used to remove the nonadherent platelets. The adhered platelets were fixed in $2.5 \%$ glutaraldehyde solution for $1 \mathrm{~h}$ at room temperature followed by dehydration in a gradient ethanol/distilled water mixture (from 50 to 100\%) for $10 \mathrm{~min}$ each. The adhered platelets onto the substrates were characterized by environmental scanning electron microscopy (AMRAY-1910FE). The platelet numbers at random fields of views for each material group were counted.

\section{Statistics analysis}

All experiments were performed at least three times, data averaged and expressed as mean \pm standard deviation. Statistical analysis was performed using one-way analysis of variance and significance was considered at $p \leq 0.05$.

\section{RESULTS AND DISCUSSION}

\section{Microstructural characterization}

Figure 1 shows the XRD patterns of all the experimental Ta samples. Typical symmetric Bragg peaks at the positions ( $2 \theta$ degree) of $38.47^{\circ}, 55.55^{\circ}, 69.58^{\circ}$, and $82.46^{\circ}$ demonstrate a well-crystallized microstructure ${ }^{17}$ of pure Ta. Because of the uniform plastic deformation, there is no peak shift or preferred orientation at the $2 \theta$ degree position compared with the initial sample. By comparison of the 0th ECAPed Ta, there is no newly formed miscellaneous peak or peak shift in the XRD patterns in the case of other Ta samples after the plastic deformation. On the basis of PDF documentation, a single phase with unique cubic structures in the featured orientation index (110), (200), (211), and (220) can be identified with typical body-centered cubic (BCC) structure, without any other phases. Thus, the group of collective glide planes and slip systems enables Ta to undertake substantial permanent deformation. The gradually broadened peaks (particularly the specific peak width at half height) observed from the corresponding XRD positions referred to the distinct grain refinement from the original 0th ECAPed Ta to the 8th ECAPed Ta based on the Scherrer equation. ${ }^{18}$ Grain size distributions and microstructural images of the experimental samples are shown in Figure 2. An average grain size of about $50 \mu \mathrm{m}$ can be calculated and analyzed in the case of the 0th ECAPed Ta sample, with recognizable equiaxed crystals and distinct grain boundaries as seen in Figure 2(b). Through heavy straining or shock loading, high-angle and nonequilibrium grain boundaries were formed and refined the grains into UFG scale by boundary sliding. After several passes treatment, the size of the grains are substantially and gradually downgraded to about $30 \pm 4.6 \mu \mathrm{m}, 10 \pm 1.1 \mu \mathrm{m}$, and $3 \pm 0.2 \mu \mathrm{m}$ for the $1 \mathrm{st}, 2 \mathrm{nd}$, and 4 th ECAPed Ta, respectively. Identified by the calculation from hundreds of single crystalline grains, the average grain size of the 8th ECAPed Ta 


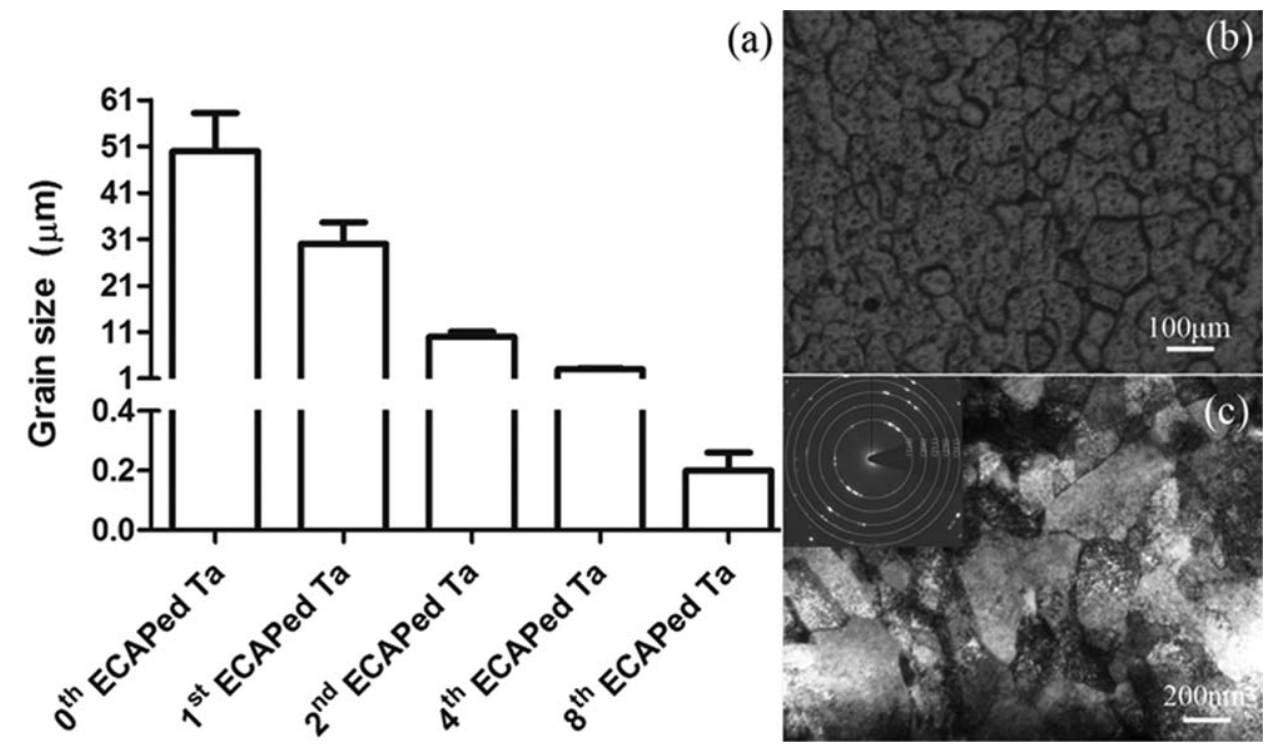

FIGURE 2. (a) Grain size of the 0th, 1st, 2nd, 4th, and 8th ECAPed Ta, and microstructural observations of SEM on (b) the 0th ECAPed Ta and TEM bright field on (c) the 8th ECAPed Ta. A plot of SAED pattern is insetted in (c).

comes to $220 \mathrm{~nm}$, as one typical TEM image shown in Figure 2(c). Moreover, the coincident positions and corresponding strength both from lumination in SAED and integral area in XRD pattern inserted in Figure 2(c) reconfirm the phases and texture structures of the 8th ECAPed Ta as an UFG Ta.

\section{Mechanical measurement}

Figure 3 shows the mechanical outcomes under the ambient temperature, including the stress-strain curves and surface microhardness of different materials. It can be clearly seen that the 0th ECAPed Ta shows a poor property in mechanical strength, ranging from approximately 100 to $200 \mathrm{MPa}$ as the yielding strength (YS; here refers to the $\sigma_{0.2}$ ) and ultimate tensile strength (UTS), respectively. However, after the stepwise deformation to certain extent, the significant strengthening effect gradually empowers due to the gradient grain size refinement. The specific parameters from the tensile loading experiment are listed in Table II. Compared to the YS of the 0th ECAPed Ta $(117.5 \pm 12.6 \mathrm{MPa})$, there is an increment of $254 \%$ in the case of the 1st ECAPed Ta $(416.1 \pm 38.9 \mathrm{MPa})$. When the grain size downgrades to an UFG scale, a much higher YS up to $754.7 \mathrm{MPa}$ is detected in the sample of the UFG 8th ECAPed Ta, over four times than that of the 0th ECAPed Ta. A similar tendency of evolutinary two- to five-folded augment can be discovered in the UTS of all the experimental samples. It is noticeable that there exists a paradox of physical strength and plasticity (elongation) of materials. It is a preconceived viewpoint that the excessive elongation or the difference between YS and UTS of pure Ta benefits their future application as coronary stents, because the nondisparity YS and UTS values will cause easy occurence of fractures during the expansion. ${ }^{19}$ The gap between YS and UTS of the ECAP-processed Ta minishes down to some extent after deformation compared to that of the originally microcrystalline pure Ta. Although the minimal difference between YS and UTS values of pure Ta of the decreased grain sizes can be found, the extremely critical/threshold value of the UFG 8th ECAPed Ta for mechanical failure/breakage makes it much difficult to get overloaded. In addition, necking occurs rapidly after the stress reaches a maximum value, yielding a uniform elongation of $5.4 \pm 0.3 \%$, which is an adequate strain value for structural metallic materials (usually regarded as $5 \%$ ) $^{20}$ and an elongation to failure of only around $2.0 \%$ occurs in the UFG 8th ECAPed Ta sample. This mechanical tradeoff is ruled by the classic dislocation-governed regime in the lattice world of metallic materials, ${ }^{21}$ nevertheless the sacrificial elongation of the UFG 8th ECAPed Ta still remains $2 \pm 0.3 \%$. Talking about the surface hardness, values in HV is documented here. HV value of the 0th ECAPed Ta is about $110 \pm 3.0 \mathrm{HV}$, which shows a good agreement with the typical conventional Ta reported elsewhere. ${ }^{22}$ After the severe deformation, the HV hardness values significantly goes up as the pass numbers increases. For the 1st ECAPed Ta, the HV value $(207 \pm 5.9)$ nearly doubles compared with that of the 0th ECAPed Ta. After two or four passes, hardness of these substrates keeps arising slightly. As for the 8th ECAPed Ta, up to three times of surface hardness $(320 \pm 6.6)$ can be demonstrated in comparison with that in the 0th ECAPed Ta. The enhanced mechanical strength as an indicative of relevant hardness can be evidently attributed as the downgrading grain size. Such a steady acceleration of hardness against deformation degree (grain size) can be well deduced by the theoretical Hall-Petch equation. ${ }^{23,24}$

\section{Electrochemical corrosion}

Figure 4 discloses the OCP curves and Tafel plots of all the Ta samples in SBF. As clearly seen within the initial $7200 \mathrm{~s}$, all the OCP curves go uprising and keep superiorly atop one 

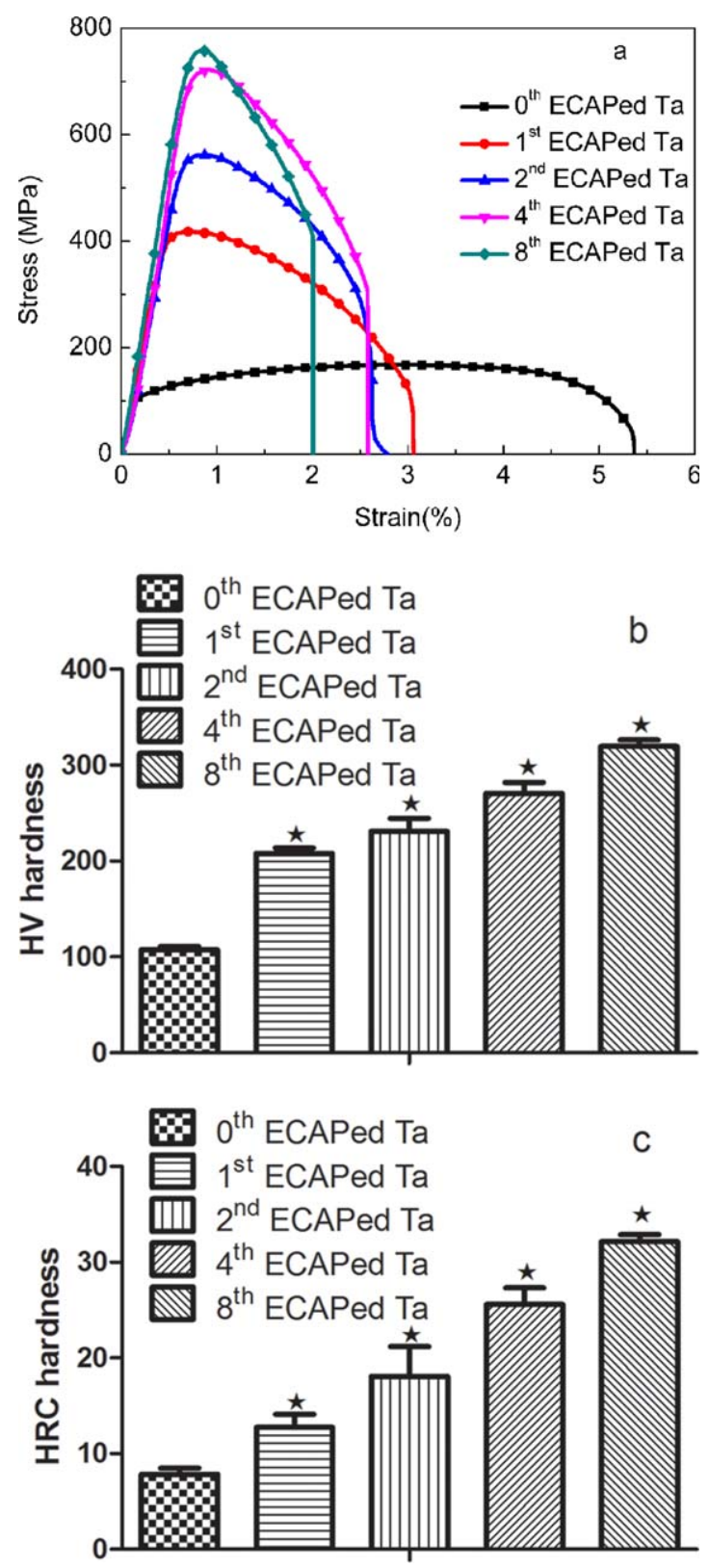

FIGURE 3. Mechanical behavior and surface hardness of the 0th, 1st, 2nd, 4th, and 8th ECAPed $\mathrm{Ta}$, (a) tensile loading curves and (b) HV plots. [Color figure can be viewed in the online issue, which is available at wileyonlinelibrary.com.]

by one as the increment of time and level of the plastic deformation, from zero deformation to 8-pass ECAP. This means an enhanced oxide layer or more stable film was favorably formed or covered on the surface of the sample with refinement structures, particularly for the UFG tex- tures. Similarly, the Tafel plot of the UFG 8th ECAPed Ta occupies the top-left position compared to that of the 4th ECAPed Ta, to say nothing of those of the 2nd, 1st, and 0th ECAPed Ta. Also analyzed from the parameters in Table III, it's easily seen that the current density $\left(i_{\text {corr }}\right)$ of the UFG 8th ECAPed Ta is the lowest $\left(0.033 \pm 0.01 \mu \mathrm{A} / \mathrm{cm}^{2}\right)$ and the corrosion potential $\left(E_{\text {corr }}\right)$ stays the highest $(-0.123 \pm$ $0.01 \mathrm{~V})$. Since the corrosion potential $(-0.468 \mathrm{~V})$ of the 0 th ECAPed Ta is known higher than that of the commercially pure Ti. Strong corrosion resistance is derived from the UFG 8th ECAPed Ta in the subtle circumstances like SBF or saline and this universally conforms to the previous discoveries of superior anticorrosion performances in the most of cases of the UFG materials (like Ti, $\mathrm{Cu}, \mathrm{Fe}$ and others). ${ }^{25-28}$ In terms of the corrosion rate described by penetration rate, the sequence of the corrosion resistance can be ordered as follows: the UFG 8th ECAPed Ta $>$ the 4th ECAPed Ta $>$ the 2nd ECAPed Ta $>$ the 1st ECAPed Ta $>$ the 0th ECAPed Ta. As for the ion release, a minimal amount of Ta ions which is lower the detection limitation of the facility can be measured in SBF until 14 days, thus the data is not listed and the threat can be omitted.

\section{Protein and cellular interactions}

To preassess the early stage of bioactivity and bioaffinity before the cellular interactions, protein attachment onto the substrates is investigated in the present study. Figure 5 shows the capability of albumin adsorption onto the experimental Ta and the TCP control within $24 \mathrm{~h}$. This plot discloses that in the first $2 \mathrm{~h}$, the protein adsorption percentage increases sharply to a level ranging from 25 to $42 \%$. Among, the 0th and 1st ECAPed Ta shows a relatively lower capacity of protein attachment (around 22\%). Median group refers to the 2nd and 4th ECAPed Ta and there is no significant difference between them. The 8th ECAPed Ta with UFG microstructure possesses a superior bioaffinity in the early stage, twice higher than the amount of the adhered protein on the substrate of microcrystalline 0th ECAPed Ta. With the passage of time, a slow yet steady growth of this percentage keeps upgoing in the follow-up durations, ending as a final peak value at around $90 \%$. During the whole period, it can be clearly seen that the percentage of the protein adsorption in the case of the UFG 8th ECAPed Ta $(42.1 \pm 3.0 \%, 56.9 \pm 4.7 \%$ and $89.0 \pm 4.1 \%$ on 2,4 , and $24 \mathrm{~h}$ incubation) leads atop over those from the TCP control $(32.2 \pm 1.8 \%, 55.4 \pm 0.3 \%$, and $84.5 \pm 2.6 \%$ on 2,4 , and $24 \mathrm{~h}$ incubation) and other Ta samples, especially for those from the microcrystalline 0th $(33.7 \pm 0.8 \%, 45.3 \pm 3.0 \%$, and $84.1 \pm 3.8 \%$ on 2 -, 4 -, and 24 -h incubation, respectively) and 1st ECAPed Ta (25.8 $\pm 3.0 \%, 49.4 \pm 4.7 \%$, and

TABLE II. Mechanical Parameters of the 0th, 1st, 2nd, 4th, and 8th ECAPed Ta

\begin{tabular}{lcccrr}
\hline & 0th ECAPed Ta & 1st ECAPed Ta & 2nd ECAPed Ta & 4th ECAPed Ta & 8th ECAPed Ta \\
\hline Yield strength (MPa) & $117.5 \pm 6$ & $416.1 \pm 28$ & $557.0 \pm 44$ & $719.0 \pm 58$ & $754.7 \pm 64$ \\
Tensile strength (MPa) & $166.0 \pm 10$ & $418.3 \pm 32$ & $561.3 \pm 47$ & $721.1 \pm 62$ & $758.9 \pm 66$ \\
Elongation (\%) & $5.4 \pm 0.3$ & $3.1 \pm 0.2$ & $2.8 \pm 0.2$ & $2.6 \pm 0.1$ & $2.0 \pm 0.1$ \\
\hline
\end{tabular}



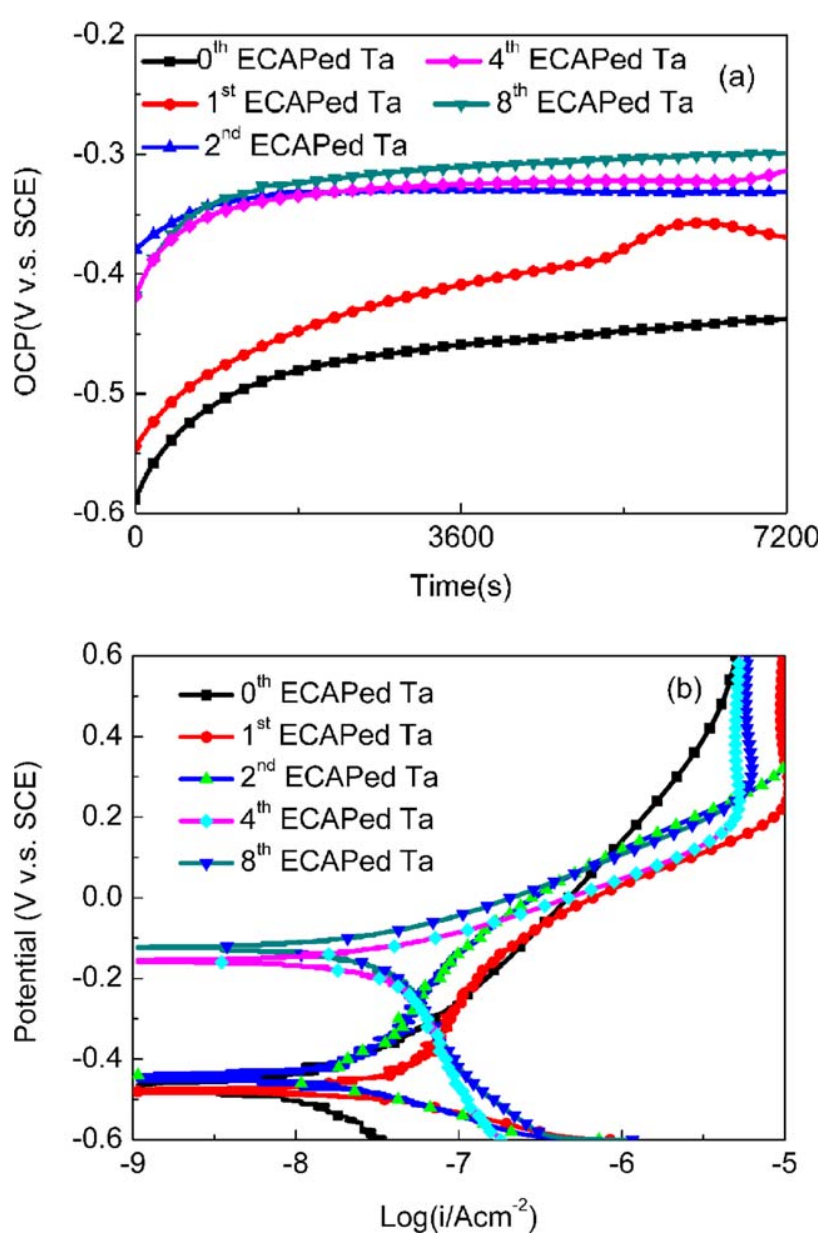

FIGURE 4. Electrochemical corrosion behavior of the 0th, 1st, 2nd, 4th, and 8th ECAPed Ta, (a) OCP curves and (b) Tafel plots. [Color figure can be viewed in the online issue, which is available at wileyonlinelibrary.com.]

$81.6 \pm 4.1 \%$ on $2-, 4-$, and $24-\mathrm{h}$ incubation, respectively) in a significant way. The favorable protein attachment of UFG Ta gives a good sign of bioaffinity all the way and this may be explained as the physiochemical difference on the surface due to the UFG framework even the nanostructure nature. ${ }^{29}$

Cell lines of murine fibroblast (L929), VSMCs and ECV304 are cultured and cell proliferation is measured in an indirect way via MTT assay in Figure 6. Figure 6(a) shows the growth curves of L929 within 4 days incubation. On the first day, cell proliferates more or less among the group of negative control and all the experimental Ta samples, with the viability ratio all above $84.2 \%$. During the following days, the group of Ta with refined grains, especially

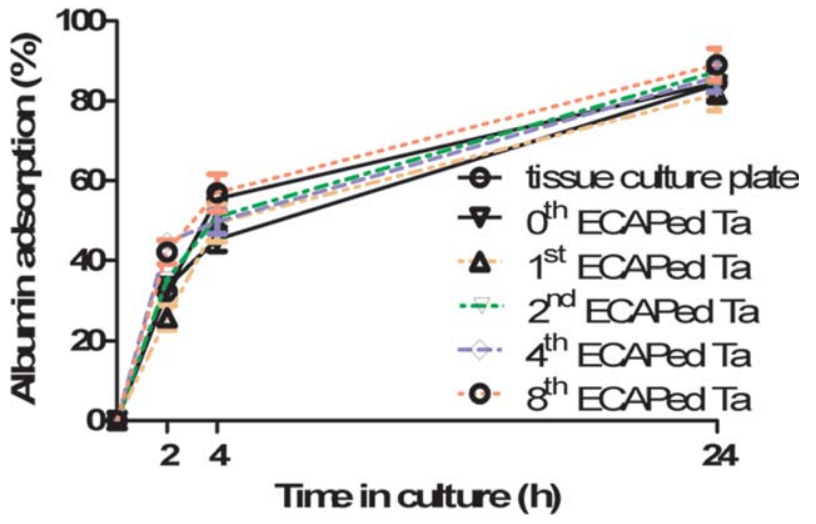

FIGURE 5. Albumin adsorption of all experimental Ta samples and the TCP control. [Color figure can be viewed in the online issue, which is available at wileyonlinelibrary.com.]

for the 8th ECAPed Ta with UFG microstructures, shows a significantly $(P=0.0362)$ pronounced cell proliferation in comparison with that from the negative control. The cell viability percentage for the UFG 8th ECAPed Ta over 100\% (112.8 and $118.7 \%$ in the 2 nd and 4 th day, respectively) implies 0-level cytotoxicity, ${ }^{30}$ which is excellent cytocompatibility without toxicity in cell growth. In terms of the VSMCs cell lines in Figure 6(b), the UFG 8th ECAPed Ta holds remarkably lower OD values compared to the negative control from the beginning till the end, although the amount of cells remains rising as time goes by. However, since the first day, the 0th ECAPed Ta keeps a higher or even similar growing level (ranging from 109.4 to $92.3 \%$ ) of the negative control. On the 2 nd and 4 th day culture, the cell proliferation reduces relatively compared to that of the negative control. The evolutionary inhibition tendency on VSMCs can be observed as the grain size decreases gradually, whereas for the growth of endothelial cells in Figure 6(c), a totally reverse principle against that in the case of VSMCs can be discovered. On the first day culture, the group of severely deformed samples such as the 2nd, 4th, and 8th ECAPed Ta manifests viability over $100 \%$ than the negative control, though not in a significant way. With the passage of time, the effect of cell promotion enlarges to $130.2 \%$ in the case of the UFG 8th ECAPed Ta on the 4th day culture.

Figure 7 showcases the morphologies of different cell lines co-cultured on different substrates in 24 h. For L929, representative shape of thinner spindle-like dots can be seen on the surface via SEM scanning. Without much cellular connections to each other, most of cell individuals were barely attached and sparsely scattered on the Ta substrates, whereas certain short pseudopods stretched up to show the activity of

TABLE III. Corrosion Parameters of the 0th, 1st, 2nd, 4th, and 8th ECAPed Ta From Tafel Plots

\begin{tabular}{lccccc}
\hline & 0th ECAPed Ta & 1st ECAPed Ta & 2nd ECAPed Ta & 4th ECAPed Ta & 8th ECAPed Ta \\
\hline$E_{\text {corr }}(\mathrm{V})$ & $-0.468 \pm 0.02$ & $-0.480 \pm 0.03$ & $-0.444 \pm 0.02$ & $-0.159 \pm 0.01$ & $-0.123 \pm 0.01$ \\
$i_{\text {corr }}\left(\mu \mathrm{A} / \mathrm{cm}^{2}\right)$ & $0.042 \pm 0.01$ & $0.039 \pm 0.01$ & $0.036 \pm 0.02$ & $0.035 \pm 0.02$ & $0.033 \pm 0.01$ \\
$R_{\mathrm{p}}\left(\mathrm{Ohm} / \mathrm{cm}^{2}\right)$ & $827041 \pm 74265$ & $912710 \pm 68954$ & $1066365 \pm 88563$ & $1149080 \pm 86954$ & $1191639 \pm 75324$ \\
Corrosion rate $\left(\mathrm{mm} / \mathrm{y}^{1}\right)$ & $0.012 \pm 0.001$ & $0.011 \pm 0.001$ & $0.011 \pm 0.001$ & $0.010 \pm 0.001$ & $0.009 \pm 0.001$ \\
\hline
\end{tabular}



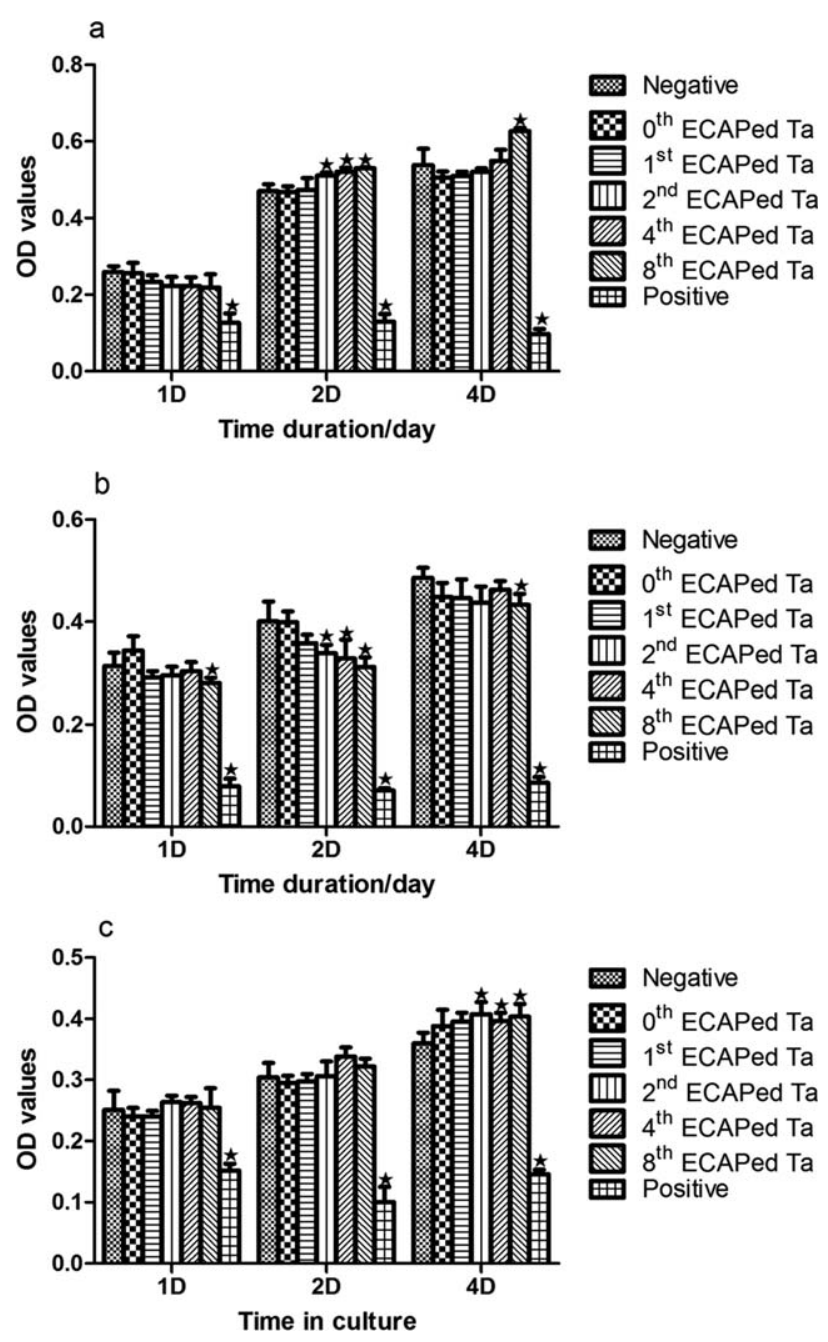

FIGURE 6. Cell responses from different cell lines: (a) murine fibroblast (L929), (b) vascular muscle smooth cells (VSMCs), and (c) endothelial cells (ECV304) with different samples.

cells, which were ready to spread out to some extent. There is no significant difference in the amount of cells among all the experimental samples, which keeps in good accordance with the MTT results in Figure 6(a). For the cases with VSMCs and ECV304, a totally contrary correlation of cell responses including numbers and morphologies status can be concluded on the different Ta substrates. Different from the tiny shape of L929, the individual sizes of elongated VSMCs and rounded ECV304 are three to five times larger than that of L929. The VSMCs look more like slender lancet and ECV304 cells appear as round leaves. In Figure 7(f), a group of VSMCs were adequately spread and mutually connected over a big coverage area on top of the 0th ECAPed Ta sample surface. In the images under higher magnification, even the cytoskeleton and spindle fibers around the nuclei could be distinguished very well. Much less amount of cell community and inferior cellular connections can be found on the substrates of the other Ta as the grain size decreases, leaving only very few cells attached on the UFG 8th ECAPed Ta in the observed area in Figure $7(j)$. The size shrinkage and vague intracellular matrix zone of these cells imply the prohibited interactions between the UFG 8th ECAPed Ta substrate and VSMCs. As for ECV304, the leaf-like cells keep their well-spread morphologies and positive growing activity on the surface of the 0th ECAPed Ta in Figure $7(\mathrm{k})$. When in contact with the refined microstructures in the 4th and 8th ECAPed Ta [as seen in Figure $7(\mathrm{n})$ and (o)], a large group of cell aggregation becomes obvious and the activity of cells can be also detected due to the undergoing fission of multinuclear cells. It's also a good indicator of grain size-based substrates from different samples contributing to the cell propagation and adhesion.

\section{Hemocompatibility evaluation}

Hemocompatibility test including hemolysis test and evaluations of the adhered platelets was also evaluated in the present study. Figure 8(a) shows that all measured hemolysis rates of experimental samples stand less than $1 \%$, way below the safety-threatening threshold $5 \% .{ }^{31}$ This demonstrates that the erythrocyte does not get destroyed or aggregated when interacted with various pure Ta samples. A distinct tendency can be seen that the hemolysis decreases sharply and gradually when the pass numbers of ECAP increases, exclusive for the 0th ECAPed Ta. After the adherent platelets were fixed onto the surface and then analyzed, the number of adhered platelet is shown in Figure 8(b). There is a relatively high density of adhered platelets on the substrates of the 0th and 1st ECAPed Ta, respectively. Whereas for those samples with refinement of grain sizes in the 2nd, 4th, and 8th ECAPed Ta, the numbers of adhered platelets remarkably declined compared to that on the 0th ECAPed Ta. As for the morphologies of the adhered platelets [Figure $8(\mathrm{c}-\mathrm{g})$ ], certain clusters of platelets with flatten shape and pseudopods as a symbol of activity can be clearly seen on the substrate of the 0th ECAPed Ta. As the amount decreases, the inactived round-shaped platelet was individually separated and scattered on the substrate of the 4th and 8th ECAPed Ta. Similarly, a gradient outcome is revealed based on the variation of microstructures and grain sizes.

\section{CONCLUSIONS}

The bulk UFG Ta was successfully fabricated via ECAP technique after till eight passes. A single BCC microstructure with the average grain size of approximate $220 \mathrm{~nm}$ is identified in the UFG 8th ECAPed Ta. A tendency of enhanced mechanical strength with applicable engineering elongation is revealed as the grain size decreases to ultrafine scale, especially for those ultrafine grains in the UFG 8th ECAPed Ta. Because of the integrated UFG nature and deficiency-free bulk induced by top-down SPD method, the 8th ECAPed Ta balanced and benefited the biological interactions in such following ways. Bulk nanostructured Ta shows excellent cytocompatibility with L929 cell lines, whereas a preferential phenomenon of prohibition growth in VSMCs and promotion in ECV304 can be found in the case of the UFG 8th ECAPed Ta. An evolutionary discipline of reduced hemolysis rate and the number and status of the adherent platelets with the downgrade of grain size can also be concluded to 


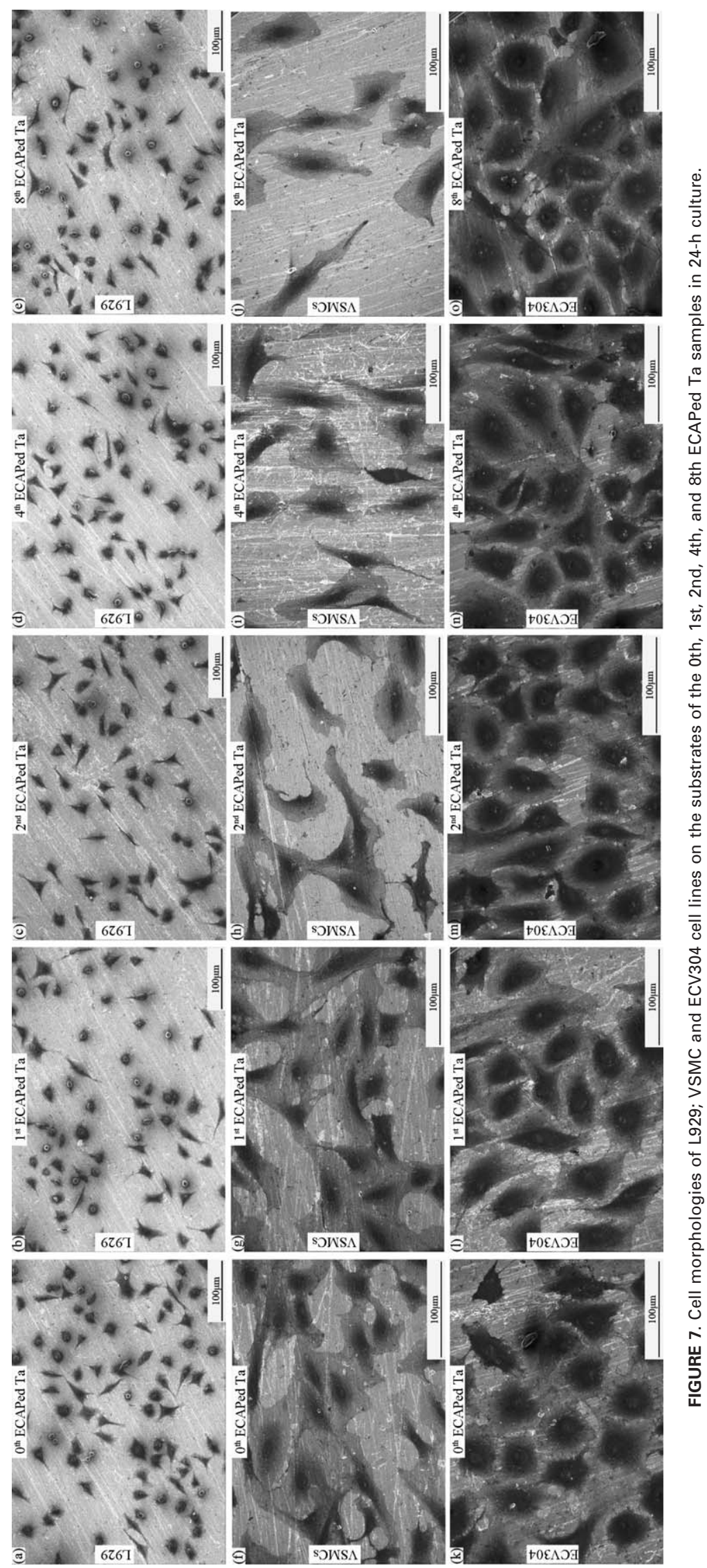



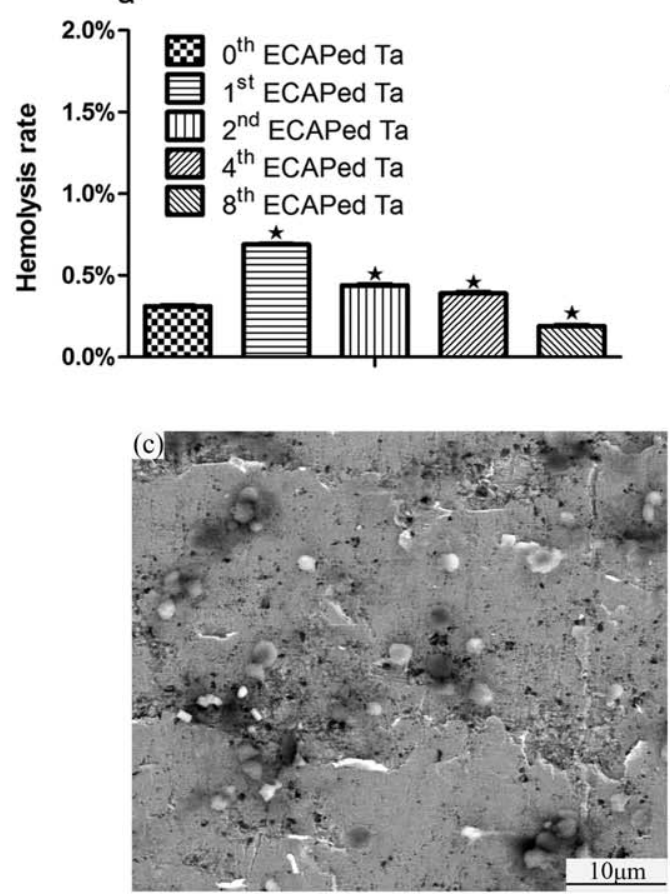

b
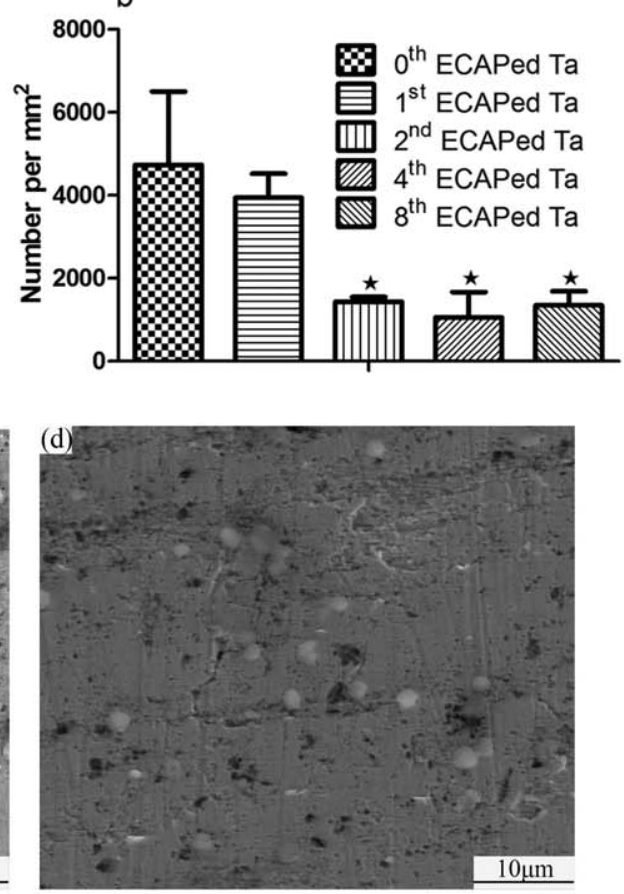
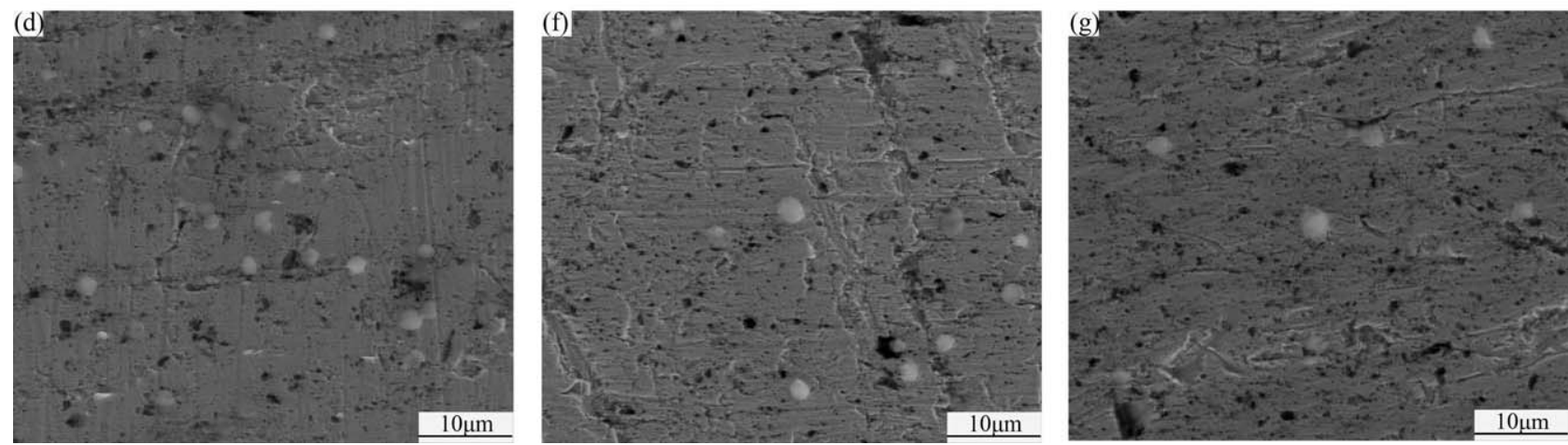

FIGURE 8. Hemocompatibility outcome of (a) hemolysis rate and (b) the amount of adhered platelets with their morphologies on the substrate of the (c) 0th; (d) 1st; (e) 2nd; (f) 4th; and (g) 8th ECAPed Ta.

show an accelerated hemocompatibility of the UFG ECAPed $\mathrm{Ta}$, which would play an alternative part in surgical or grafting field.

\section{REFERENCES}

1. ASTM-F560-92: Standard Specification for Unalloyed Tantalum for Surgical Implant Applications. Philadelphia: American Society for Testing and Materials; 1993;13.01. pp 92-93.

2. Black J. Biological performance of tantalum. Clin Mater 1994;16: 167-173.

3. Zitter H, Plenk H Jr. The electrochemical behavior of metallic implant materials as an indicator of their biocompatibility. J Biomed Mater Res 1987;21:881-896.

4. Mani G, Feldman MD, Patel D, Agrawal CM. Coronary stents: A materials perspective. Biomaterials 2007;28:1689-1710.

5. Miyazaki T, Kim HM, Kokubo T, Ohtsuki C, Kato H, Nakamura T. Mechanism of bonelike apatite formation on bioactive tantalum metal in a simulated body fluid. Biomaterials 2002;23:827-832.

6. Bobyn JD, Stackpool GJ, Hacking SA, Tanzer M, Krygier JJ. Characteristics of bone ingrowth and interface mechanics of a new porous tantalum biomaterial. J Bone Joint Surg 1999;81B:5-9.
7. Bobyn JD, Toh KK, Hacking SA, Tanzer M, Krygier JJ. Tissue response to porous tantalum acetabular cups. J Arthroplasty 1999;3:347-354.

8. Sharma CP, Paul W. Protein interaction with tantalum: Changes with oxide layer and hydroxyapatite at the surface interface. J Biomed Mater Res 1992;26:1179-1184.

9. Chen JY, Leng YX, Tian XB, Wang LP, Huang N, Chu PK, Yang P. Antithrombogenic investigation of surface energy and optical bandgap and hemocompatibility mechanism of $\mathrm{Ti}\left(\mathrm{Ta}^{+5}\right) \mathrm{O}_{2}$ thin films. Biomaterials 2002;23:2545-2552.

10. Kato H, Nakamura $T$, Nishiguchi $S$, Matsusue $Y$, Kobayashi M, Miyazaki T, Kim HM, Kokubo T. Bonding of alkali- and heattreated tantalum implants to bone. J Biomed Mater Res 2000;53: 28-35.

11. Crouchet D, Grossetete R, Bach-Lijour B, Sagan C, Lecomte $E$, Leurent B, Brunel P, Le Nihouannen JC. Plasma treatment effects on the tantalum strecker stent implanted in femoral arteries of sheep. Cardiovasc Intervent Radiol 1994;17:285-291.

12. Johnson PF, Bernstein JJ, Hunter G, Dawson WW, Hench LL. An in vitro and in vivo analysis of anodized tantalum capacitive electrodes: Corrosion response, physiology and histology. J Biomed Mater Res 1977;11:637-656. 
13. Stolyarov VV, Zhu YT, Lowe TC, Islamgaliev RK, Valiev RZ. A two step SPD processing of ultrafine-grained titanium. Nanostruct Mater 1999:7:947-954.

14. ASTM-G31-72. Standard Practice for Laboratory Immersion Corrosion Testing Of Metals. Annual book of ASTM standards. Philadelphia: American Society for Testing and Materials; 2004.

15. Zhang EW, Wang YB, Gao F, Wei SC, Zheng YF. Enhanced bioactivity of sandblasted and acid-etched titanium surfaces. Adv Mater Res 2009;79-82:393-396.

16. Gu XN, Zheng YF, Cheng $Y$, Zhong SP, Xi TF. In vitro corrosion and biocompatibility of binary $\mathrm{Mg}-1 \mathrm{wt} \% \mathrm{X}$ alloys. Biomaterials 2009;30:484-498.

17. Bayon R, Herrero J. Structure and morphology of the indium hydroxy sulphide thin films. Appl Surf Sci 2000;158:49-57.

18. Jenkins R, Snyder RL. Introduction to X-ray Powder Diffractometry. John Wiley \&\#38; Sons Inc.; 1996. pp 89-91.

19. Liu B, Zheng YF. Effects of alloying elements (Mn, Co, Al, W, Sn, $\mathrm{B}, \mathrm{C}$ and S) on biodegradability and in vitro biocompatibility of pure iron. Acta Biomaterialia 2011;7:1407-1420.

20. Zhao $Y H$, Liao $X Z$, Cheng $S$, Ma E, Zhu YT. Simultaneously increasing the ductility and strength of nanostructured alloys. Adv Mater 2006;18:2280-2283.

21. Valiev RZ. Nanostructuring of metals by severe plastic deformation for advanced properties. Nat Mater 2004;3:511-516.

22. Mimura K, Nanjo M. Production of pure tantalum by carbonreduction smelting and hydrogen plasma-arc melting with refining. Mater Trans JIM 1990;4:293-301.
23. Sylwestrowicz $W$, Hall EO. The deformation and ageing of mild steel. Proc Phys Soc Sect B 1951;64:495-502.

24. Petch N. The cleavage strength of polycrystals. J Iron Steel Inst 1953;174:25-28.

25. Balyanov A, Kutnyakova J, Amirkhanova NA, Stolyarov VV, Valive RZ, Liao XZ, Zhao YH, Jiang YB, Xu HF, Lowe TC, Zhu YT. Corrosion resistance of ultra fine-grained Ti. Scr Materialia 2004;51: 225-229.

26. Vinogradov A, Mimaki T, Hashimoto S, Valiev R. On the corrosion behavior of ultra-fine grain copper. Scr Materialia 1999;41: 319-326.

27. Nie FL, Zheng YF, Wei SC, Hu C, Yang G. In vitro corrosion, cytotoxicity and hemocompatibility of bulk nanocrystalline pure iron. Biomed Mater 2010;5:1-11.

28. Nie FL, Zheng YF. Surface chemistry of bulk nanocrystalline pure iron and electrochemistry study in gas-flow physiological saline. J Biomed Mater Res B Appl Biomater 2012;100: 1404-1410.

29. Tran PA, Sarin L, Hurt RH, Webster TJ. Opportunities for nanotechnology-enabled bioactive bone implants. J Mater Chem 2009;19:2653-2659.

30. ISO 10993-5. Biological Evaluation of Medical Devices. Part 5: Tests for In Vitro Cytotoxicity. Geneva: International Organization for Standardization; 1999.

31. ISO 10993-4. Biological Evaluation of Medical Devices. Part 4: Selection of Tests for Interactions With Blood. Geneva: International Organization for Standardization; 2002. 